\title{
Target and resistance-related proteins of recombinant mutant human tumor necrosis factor-related apoptosis-inducing ligand on myeloma cell lines
}

\author{
YUAN JIAN $^{1}$, YULING CHEN ${ }^{2}$, CHUANYING GENG ${ }^{1}$, NIAN LIU ${ }^{1}$, \\ GUANGZHONG YANG ${ }^{1}$, JINWEI LIU ${ }^{1}$, XIN LI $^{1}$, HAITENG DENG $^{2 *}$ and WENMING CHEN ${ }^{1 *}$ \\ ${ }^{1}$ Department of Hematology, Beijing Chao-yang Hospital, Capital Medical University, Beijing 100020; \\ ${ }^{2}$ Ministry of Education Key Laboratory of Bioinformatics, School of Life Sciences, \\ Tsinghua University, Beijing 100084, P.R. China
}

Received December 7, 2015; Accepted April 6, 2016

DOI: 10.3892/br.2016.650

\begin{abstract}
Recombinant mutant human tumor necrosis factor-related apoptosis-inducing ligand (rmhTRAIL) has become a potential therapeutic drug for multiple myeloma (MM). However, the exact targets and resistance mechanisms of rmhTRAIL on MM cells remain to be elucidated. The present study aimed to investigate the target and resistance-related proteins of rmhTRAIL on myeloma cell lines. A TRAIL-sensitive myeloma cell line, RPMI 8226, and a TRAIL-resistance one, U266, were chosen and the differentially expressed proteins between the two cell lines were analyzed prior and subsequent to rmhTRAIL administration by a liquid chromatography-tandem mass spectrometry method. The results showed that following TRAIL treatment, 6 apoptosis-related proteins, calpain small subunit 1 (CPNS1), peflin (PEF1), B-cell receptor-associated protein 31 (BAP31), apoptosis-associated speck-like protein containing CARD (ASC), BAG family molecular chaperone regulator 2 (BAG2) and chromobox protein homolog 3 (CBX3), were upregulated in RPMI 8226 cells while no change was identified in the U266 cells. Furthermore, small ubiquitin-related modifier 1 and several other ubiquitin proteasome pathway (UPP)-related proteins expressed higher levels in TRAIL-resistant cells
\end{abstract}

Correspondence to: Professor Haiteng Deng, Ministry of Education Key Laboratory of Bioinformatics, School of Life Sciences, Tsinghua University, Beijing 100084, P.R. China

E-mail: dht@mail.tsinghua.edu.cn

Professor Wenming Chen, Department of Hematology, Beijing Chao-yang Hospital, Capital Medical University, 8 Gongti Nanlu Road, Beijing 100020, P.R. China

E-mail: 13910107759@163.com

*Contributed equally

Key words: multiple myeloma, recombinant mutant human tumor necrosis factor-related apoptosis-inducing ligand, proteomics, target protein, resistant-related protein
U266 compared to the RPMI-8226 cells prior and subsequent to rmhTRAIL treatment. These results suggested that CPNS1, PEF1, BAP31, ASC, BAG2 and CBX3 were possibly target proteins of rmhTRAIL on RPMI 8226 cells, while UPP may have a vital role in mediating TRAIL-resistance in U266 cells.

\section{Introduction}

Multiple myeloma (MM) is a clonal plasma cell malignancy characterized by bone, renal, hematological and often neurological complications (1). Although the response rate of MM patients has significantly improved over the last decade due to the broad use of novel agents, the majority of patients will eventually succumb due to complications associated with the development of resistant disease (2). There remains a requirement for new therapeutic options for patients with this B-cell malignancy. Tumor necrosis factor-related apoptosis-inducing ligand (TRAIL) has become a potential therapeutic drug for MM, as it selectively induces apoptosis in various types of tumor cells, including myeloma cells, while it shows no significant untoward effects on normal cells $(3,4)$. Recombinant mutant human TRAIL (rmhTRAIL) is optimized from wild-type TRAIL. It has significant improvements in terms of stability, solubility and biological activity (5).

It has been found that the sensitivity of myeloma cells to TRAIL varies considerably in vitro and in vivo. However, the exact targets and resistance mechanisms of TRAIL on MM cells are controversial. Certain studies have addressed that TRAIL has a specific apoptosis-inducing effect in tumor cells by combining with TRAIL receptors on cell membranes (6-8). By contrast, certain studies argue that the sensitivity of MM cells to TRAIL has no reference to the level of TRAIL receptors $(9,10)$. To determine whether there are other targets and resistance mechanisms of TRAIL on MM cells, the differentially expressed proteins were compared between TRAIL-sensitive and TRAIL-resistant cell lines prior and subsequent to rmhTRAIL administration by a global proteomic-based approach, and the promising target and resistance-related proteins were analyzed. 


\section{Materials and methods}

Reagents and cell lines. RmhTRAIL freeze-dried powder (Beijing Sunbio Biotech Co., Ltd., Beijing, China) was diluted in distilled water to a $1 \mathrm{mg} / \mathrm{ml} \mathrm{rmhTRAIL} \mathrm{solution,} \mathrm{and} \mathrm{was}$ preserved and protected from air at $-20^{\circ} \mathrm{C}$ in aliquots, and diluted to a working concentration in RPMI-1640 prior to use. The human myeloma cell line RPMI 8226 (Beijing Sunbio Biotech Co., Ltd.) and U266 (Cancer Institute and Hospital, Chinese Academy of Medical Sciences, Beijing, China) were cultured in RPMI-1640 supplemented with $1 \%$ penicillin/streptomycin, $1 \mathrm{mmol} / 1 \mathrm{~L}$-glutamine and $10 \%$ fetal bovine serum at $37^{\circ} \mathrm{C}, 5 \% \mathrm{CO}_{2}$ in air.

TRAIL treatment. RPMI 8226 and U266 cells $\left(2 \times 10^{7}\right.$ cells each) were treated with 15.625 and $1,000 \mathrm{ng} / \mathrm{ml} \mathrm{rmhTRAIL}$, respectively, for $24 \mathrm{~h}$ (8226TRAIL and U266TRAIL), and untreated cells were used as the control (8226CON and U266CON). The concentrations were selected as the apoptosis ratios were 4.29 and $0.54 \%$, respectively, according to our previous study (unpublished data).

One-dimensional sodium dodecyl sulfate-polyacrylamide gel electrophoresis (1D-SDS-PAGE). Following treatment, the cells were washed three times with phosphate-buffered saline. Total proteins from the four groups of cells were extracted from cells using the Total Protein Extraction kit (Beijing Biosynthesis Biotechnology Co., Ltd., Beijing, China). Cellular debris was removed by centrifugation for $30 \mathrm{~min}$ at $13,000 \mathrm{xg}$ and at $4^{\circ} \mathrm{C}$. Protein concentrations were determined using the bicinchonininc acid (Beijing Biosynthesis Biotechnology Co., Ltd.) assay. Protein samples were separated by $12 \%$ 1D-SDS-PAGE and stained with Coomassie Brilliant Blue R250 solution (Beijing Biosynthesis Biotechnology Co., Ltd.). Protein zones were manually excised from the gels. Subsequently, gel sections were destained and dehydrated with acetonitrile.

Sample preparation. Subsequent to the samples being destained and dehydrated, the proteins in the gel sections were reduced with dithiothreitol, alkylated with iodoacetamide and incubated with $12.5 \mu \mathrm{g} / \mu \mathrm{l}$ sequencing grade trypsin (Promega, Madison, WI, USA) at $37^{\circ} \mathrm{C}$ for $12 \mathrm{~h}$. For protein quantification, peptides of the four groups of cells were labeled with disparate $\mathrm{TMT}^{6}$ reagents (Pierce Biotechnology, Thermo Fisher Scientific, Inc., Rockford, IL, USA). In detail, the U266TRAIL group was labeled with TMT $^{6}-128$, U266CON group with TMT ${ }^{6}-129,8226 \mathrm{TRAIL}$ group with $\mathrm{TMT}^{6}-130$ and $8226 \mathrm{CON}$ group with $\mathrm{TMT}^{6}-131$ respectively, according to the manufacturer's protocol. The labeling reaction was carried out by incubation of tryptic peptides with the TMT reagents for $1 \mathrm{~h}$ at room temperature, and was quenched by hydroxylamine. The TMT-labeled peptides were desalted using the stage tips. Following the labeling, the peptides were extracted with $0.1 \%$ formic acid, and dried in a vacuum centrifuge. The volumes of the extraction were adjusted to $25 \mu \mathrm{l}$ with $0.1 \%$ trilfluoroacetic acid, of which $20 \mu \mathrm{l}$ was analyzed by liquid chromatography-tandem mass spectrometry (LC-MS/MS).

$L C-M S / M S$. For LC-MS/MS analysis, each digestion product was separated by a 65 -min gradient elution at a flow rate
$0.25 \mu 1 / \mathrm{min}$ with the EASY-nLCII ${ }^{\mathrm{TM}}$ integrated nano-HPLC system (Proxeon Biosystems A/S, Odense C, Denmark), which is directly interfaced with the Thermo LTQ-Orbitrap mass spectrometer. The LTQ-Orbitrap mass spectrometer was operated in the data-dependent acquisition mode using the Xcalibur software (Thermo Fisher Scientific, Inc.). The experiment consisted of a single full-scan mass spectrum in the Orbitrap (400-1,800 m/z, 30,000 resolutions) followed by 20 data-dependent MS/MS scans in the ion trap at $35 \%$ normalized collision energy. The MS/MS spectra from each LC-MS/MS run were searched against the selected database using an in-house Proteome Discovery searching algorithm (10).

Bioinformatics analysis of proteins. The MS/MS peak lists were searched against the IPI human database using SEQUEST software (http://fields.scripps.edu/sequest/). The search criteria were as follows: Full tryptic specificity was required; one missed cleavages were allowed; carbamidomethylation was set as fixed modification; the oxidation was set as the variable modification; precursor ion mass tolerances were set at $10 \mathrm{ppm}$ for all MS acquired in the Orbitrap mass analyzer; and the fragment ion mass tolerance was set at $0.8 \mathrm{Da}$ for all MS2 spectra acquired in the linear ion trap (11). The peptides data were further filtered by selecting proteins with ProtScore $>2$ and at least two unique peptides. Cut-off TMT ratios of fold-change for protein expression were $>1.5$ for upregulation and $<0.67$ for downregulation.

Differentially expressed proteins were classified based on the Protein ANalysis THrough Evolutionary Relationships (PANTHER) system (http://www.pantherdb.org), which is a unique resource that classifies genes and proteins by their functions $(12,13)$. Certain proteins were annotated manually based on literature searches and closely associated homologues.

The differentially expressed protein interaction networks were built automatically by the Search Tool for the Retrieval of Interacting Genes/Proteins (STRING) system (http://string-db. org) with the default setting, except that organism, confidence (score) and interactors shown were set to 'human', '0.20', and 'no more than 10 interactors', respectively $(14,15)$. The gene name list of these proteins was input to search against the database, which contains known and predicted protein-protein interactions. The retrieve included a detailed network, which highlights several hub proteins.

\section{Results}

Proteome profiles of rmhTRAIL-treated and control myeloma cells. Four groups of proteins were separated in SDS-PAGE gels, which were subsequently stained with Coomassie Brilliant Blue R250. Each of the four stained polyacrylamide gels was divided into 10 sections (Fig. 1).

LC-MS/MS identification revealed that 1,594 proteins were identified, which had a ProtScore $>2$ and at least two unique peptides. $42 \%$ (675 of 1,594) proteins were identified by $>5$ peptides, $12 \%$ ( 190 of 1,594$)$ by 4 peptides, $17 \%$ (278 of 1,594$)$ by 3 peptides and $28 \%$ (451 of 1,594) by 2 peptides.

Target proteins of rmhTRAIL on RPMI 8226 cells. Among the total 1,594 proteins, 337 differentially expressed proteins were 
Table I. Target proteins of rmhTRAIL on RPMI8226 cells according to liquid chromatography-tandem mass spectrometry identification.

\begin{tabular}{|c|c|c|c|c|c|}
\hline $\mathrm{Name}^{\mathrm{a}}$ & Function & Accession $^{b}$ & Score $^{c}$ & $\begin{array}{l}\text { 8226TRAIL/ } \\
8226 \mathrm{CON}^{\mathrm{d}}\end{array}$ & $\begin{array}{l}\text { U266TRAIL/ } \\
\text { U266CON }^{\mathrm{e}}\end{array}$ \\
\hline $\begin{array}{l}\text { Calpain small } \\
\text { subunit } 1 \text { (CPNS1) }\end{array}$ & $\begin{array}{l}\text { Induction of apoptosis; } \\
\text { immune system process; } \\
\text { proteolysis }\end{array}$ & IPI00025084 & 37.23 & 1.941 & 0.800 \\
\hline Peflin (PEF1) & $\begin{array}{l}\text { Induction of apoptosis; } \\
\text { immune system process; } \\
\text { proteolysis }\end{array}$ & IPI00018235 & 30.84 & 1.646 & 1.021 \\
\hline $\begin{array}{l}\text { B-cell receptor-associated } \\
\text { protein } 31 \text { (BAP31) }\end{array}$ & $\begin{array}{l}\text { Apoptosis; } \\
\text { intracellular protein transport }\end{array}$ & IPI00218200 & 18.53 & 1.958 & 0.831 \\
\hline $\begin{array}{l}\text { Isoform } 2 \text { of apoptosis-associated } \\
\text { speck-like protein } \\
\text { containing a CARD (ASC) }\end{array}$ & $\begin{array}{l}\text { Apoptosis; } \\
\text { proteolysis }\end{array}$ & IPI00221360 & 17.16 & 1.925 & 0.731 \\
\hline $\begin{array}{l}\text { BAG family molecular } \\
\text { chaperone regulator } 2 \text { (BAG2) }\end{array}$ & $\begin{array}{l}\text { Apoptosis; } \\
\text { protein folding }\end{array}$ & IPI00000643 & 11.29 & 1.599 & 0.822 \\
\hline $\begin{array}{l}\text { Chromobox protein } \\
\text { homolog } 3(\mathrm{CBX} 3)\end{array}$ & $\begin{array}{l}\text { Apoptosis; } \\
\text { regulation of transcription from } \\
\text { RNA polymerase II promoter; } \\
\text { establishment or maintenance } \\
\text { of chromatin }\end{array}$ & IPI00297579 & 7.47 & 2.303 & 0.929 \\
\hline
\end{tabular}

aProtein description in the IPI human database. ${ }^{\mathrm{b}}$ Accession number in the IPI human database. ${ }^{\mathrm{c}}$ Level at which the peptide matches the protein. ${ }^{\mathrm{d}}$ Ratio of proteins in the 8226TRAIL/8226CON pair. ${ }^{\mathrm{e}}$ Ratio of proteins in the U266TRAIL/U266CON pair. rmhTRAIL, recombinant mutant human tumor necrosis factor-related apoptosis-inducing ligand; CON, control.

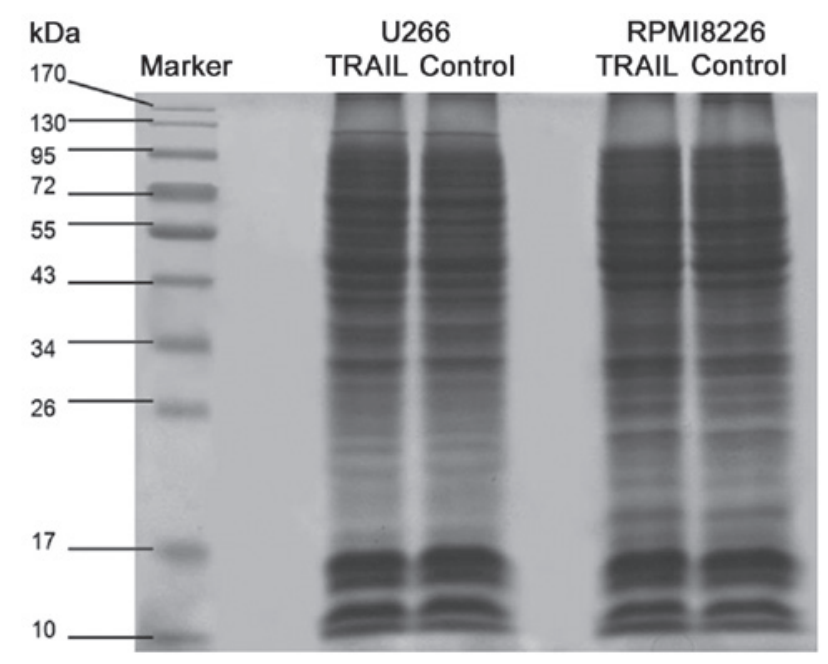

Figure 1. Sodium dodecyl sulfate-polyacrylamide gel electrophoresis (12\%) of the proteins from the four treatment groups. TRAIL, tumor necrosis factor-related apoptosis-inducing ligand.

screened in $8226 \mathrm{TRAIL} / 8226 \mathrm{CON}$ pair, while 757 differentially expressed proteins in U266TRAIL/U266CON pair, among which 245 and 492 proteins could be recognized and analyzed by PANTHER. These proteins were involved in various biological processes (Fig. 2).

Differentially expressed proteins of the two pairs were further analyzed, and the proteins that participated in apoptosis were screened by PANTHER. Among them, 6 pro-apoptotic proteins were screened as possible target proteins of rmhTRAIL on RPMI 8226 cells (Table I), which were upregulated in the 8226TRAIL/8226CON pair, while no change was observed in the U266TRAIL/U266CON pair. These were calpain small subunit 1 (CPNS1), peflin (PEF1), B-cell receptor-associated protein 31 (BAP31), apoptosis-associated speck-like protein containing CARD (ASC), BAG family molecular chaperone regulator 2 (BAG2) and chromobox protein homolog 3 (CBX3), respectively.

Resistance-related proteins of rmhTRAIL on U266 cells. Among the total 1,594 proteins, 1,081 differentially expressed proteins were screened in the U266CON/8226CON pair, while 662 differentially expressed proteins were in the U266TRAIL/8226TRAIL pair, among which 702 and 450 proteins could be recognized and analyzed by PANTHER. The differentially expressed proteins of the two pairs were further analyzed, and the proteins that participated in apoptosis or proliferation were screened by PANTHER. Data showed that there were 31 proliferation- or anti-apoptosis-related proteins expressed at higher levels in the TRAIL-resistant cells U266 compared to RPMI 8226 cells prior and subsequent to rmhTRAIL treatment. Among the 31 identified proteins, 20 can be linked through direct interaction into a protein-protein interaction network based on the prediction results of STRING system (Fig. 3). Notably, small ubiquitin-related modifier 1 (SUMO1) and several other proteins, which participated in the ubiquitin proteasome pathway (UPP), were hubs in this network. Of the 20 proteins, 


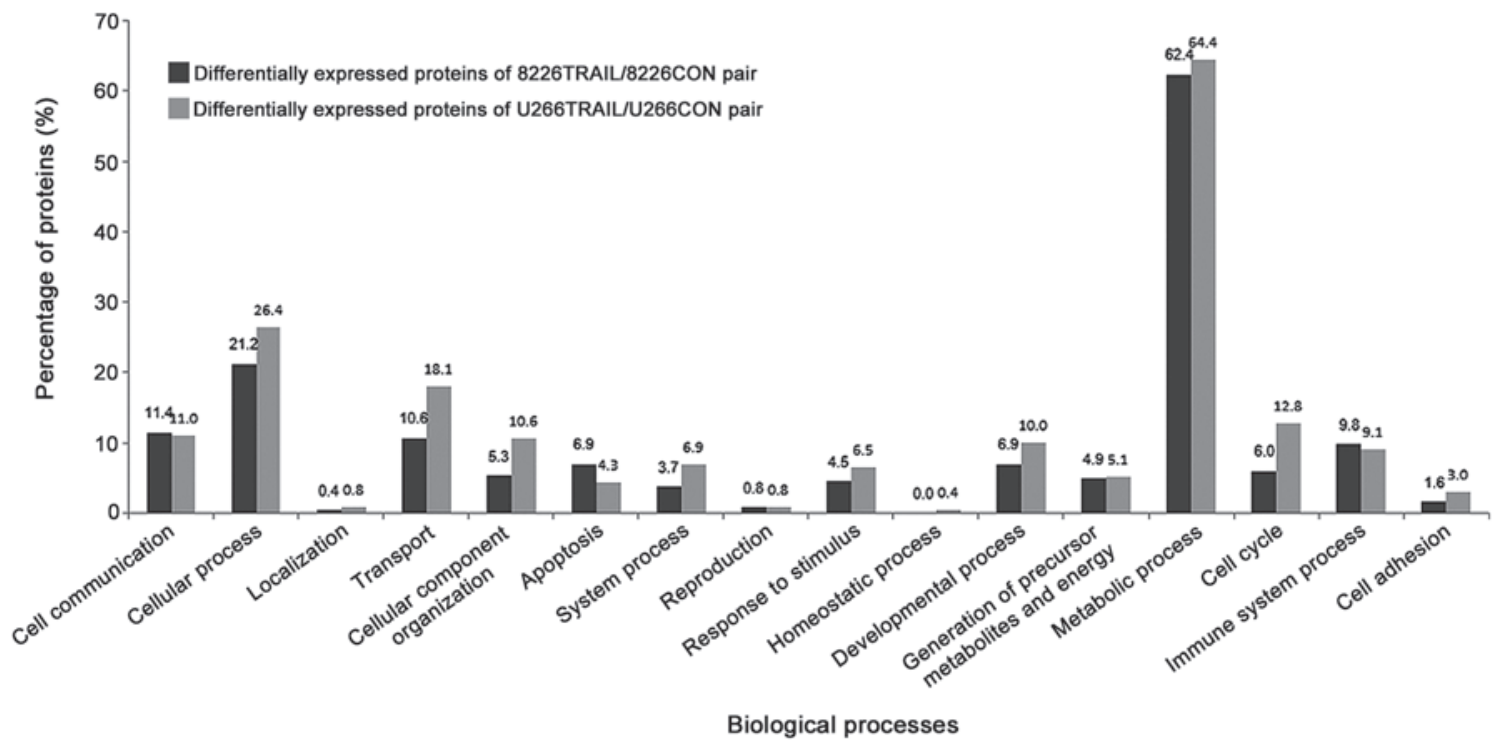

Figure 2. Biological processes of the differentially expressed proteins of the 8226TRAIL/8226CON and U266TRAIL/U266CON pairs. TRAIL, tumor necrosis factor-related apoptosis-inducing ligand.

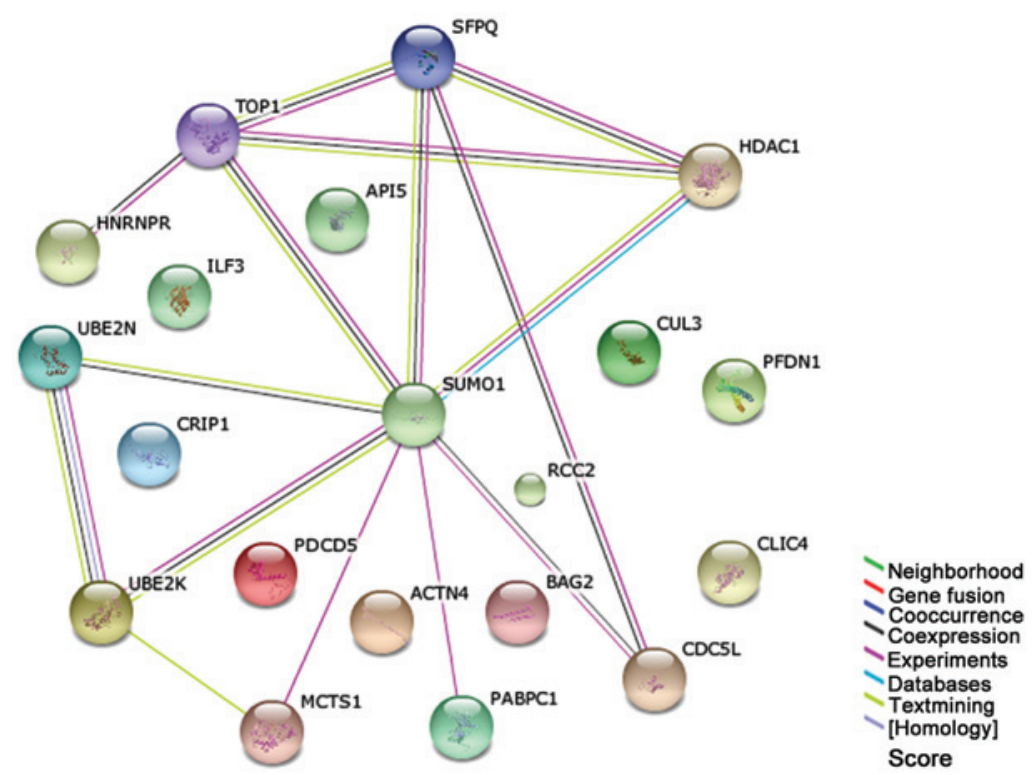

Figure 3. Protein-protein interaction network of the identified proteins. The network containing 20 identified proteins was mapped using the STRING system (http://string-db.org/) based on evidence with different types. In the evidence view, the links between proteins represent possible interactions. Different line colors represent the types of evidence for the associations, which are shown in the legend.

12 are known SUMO1 interactors. These results showed that UPP proteins were overexpressed in U266 cells and were independent of rmhTRAIL treatment, suggesting that UPP may have a vital role in mediating TRAIL-resistance in U266 cells.

\section{Discussion}

The present study analyzed the TRAIL-targeted proteins and the resistance-related proteins by a global proteomic-based approach. According to the results, the possible target proteins of rmhTRAIL on RPMI 8226 cells were CPNS1, PEF1, BAP31, ASC, BAG2 and CBX3. The possible resistance mechanism of rmhTRAIL on U266 cells was the overexpression of UPP-related proteins.
To date, studies in MM show that TRAIL induces cell apoptosis via the extrinsic and intrinsic pathways (16). According to this view, TRAIL receptors are considered essential in these two approaches, which is inconsistent with certain studies that argue the sensitivity of MM cells to TRAIL has no reference to the level of TRAIL receptors $(9,10)$. With the present data, the pro-apoptosis proteins CPNS1, PEF1, BAP31, ASC, BAG2 and CBX3 may be the apoptosis-induced mechanisms in addition to the activation of death receptors.

Furthermore, a previous study showed that the resistance mechanisms of myeloma cells to TRAIL contain various pathways, including variable levels of TRAIL receptors, variable expression levels of molecules participating extrinsic pathway or intrinsic pathway, and drug resistance mediated by bone 
marrow stromal cells (17). UPP is an important mechanism in the degradation of proteins $(18,19)$, which has not been reported in previous studies of TRAIL-resistance mechanism in myeloma cells. Recent studies showed that the disorder of tumor cell regulatory function can be due to the inactivation of key regulators. Therefore, the proteasome inhibitor has become an effective therapeutic approach of tumor treatment, particularly for the treatment of hematological malignancies (18). For myeloma, the proteasome inhibitor bortezomib has shown significant effectiveness and tolerance, while the synergistic effect with various drugs has been shown (20). According to the present result, the combination of TRAIL with the proteasome inhibitor may show a synergistic effect in the TRAIL-resistant cell line U266, and the resistance of U266 to TRAIL may be reversed theoretically.

As several pro- and anti-apoptosis proteins have been screened by LC-MS/MS, their functions could be identified in the following step to a more extensive verification of the proteins screened by the present study. In addition, TRAIL receptors were not identified by LC-MS/MS, which could be due to the limitation of the mass spectrum, as the proteins with a low concentration could not be identified (21).

In conclusion, the present study provides a feasible method to explore targets and resistance mechanisms for drugs and new information regarding rmhTRAIL on myeloma cells. According to the results from the global proteomic-based approach, the possible target proteins of rmhTRAIL on RPMI 8226 cells are CPNS1, PEF1, BAP31, ASC, BAG2 and CBX3. While the possible resistance mechanism of rmhTRAIL on U266 cells is the overexpression of UPP-related proteins.

\section{References}

1. Pratt G, Jenner M, Owen R, Snowden JA, Ashcroft J, Yong K, Feyler S, Morgan G, Cavenagh J, Cook G, et al: Updates to the guidelines for the diagnosis and management of multiple myeloma. Br J Haematol 167: 131-133, 2014.

2. Harousseau JL, Shaughnessy J Jr and Richardson P: Multiple myeloma. Hematology (Am Soc Hematol Educ Program) 2004: 237-256, 2004

3. Gazitt Y: TRAIL is a potent inducer of apoptosis in myeloma cells derived from multiple myeloma patients and is not cytotoxic to hematopoietic stem cells. Leukemia 13: 1817-1824, 1999.

4. Geng C, Hou J, Zhao Y, Ke X, Wang Z, Qiu L, Xi H, Wang F, Wei N, Liu Y, et al: A multicenter, open-label phase II study of recombinant CPT (Circularly Permuted TRAIL) plus thalidomide in patients with relapsed and refractory multiple myeloma. Am J Hematol 89: 1037-1042, 2014.
5. Fang F, Wang AP and Yang SF: Antitumor activity of a novel recombinant mutant human tumor necrosis factor-related apoptosis-inducing ligand. Acta Pharmacol Sin 26: 1373-1381, 2005.

6. Pan G, O'Rourke K, Chinnaiyan AM, Gentz R, Ebner R, Ni J and Dixit VM: The receptor for the cytotoxic ligand TRAIL. Science 276: 111-113, 1997.

7. Pan G, Ni J, Yu G, Wei YF and Dixit VM: TRUNDD, a new member of the TRAIL receptor family that antagonizes TRAIL signalling. FEBS Lett 424: 41-45, 1998

8. Marsters SA, Sheridan JP, Pitti RM, Huang A, Skubatch M, Baldwin D, Yuan J, Gurney A, Goddard AD, Godowski P, et al: A novel receptor for Apo2L/TRAIL contains a truncated death domain. Curr Biol 7: 1003-1006, 1997.

9. Mitsiades CS, Treon SP, Mitsiades N, Shima Y, Richardson P, Schlossman R, Hideshima T and Anderson KC: TRAIL/Apo2L ligand selectively induces apoptosis and overcomes drug resistance in multiple myeloma: Therapeutic applications. Blood 98: 795-804, 2001.

10. Gómez-Benito M, Martinez-Lorenzo MJ, Anel A, Marzo I and Naval J: Membrane expression of DR4, DR5 and caspase-8 levels, but not Mcl-1, determine sensitivity of human myeloma cells to Apo2L/TRAIL. Exp Cell Res 313: 2378-2388, 2007.

11. Hong D, Chen HX, Yu HQ, Wang C, Deng HT, Lian QQ and Ge RS: Quantitative proteomic analysis of dexamethasone-induced effects on osteoblast differentiation, proliferation, and apoptosis in MC3T3-E1 cells using SILAC. Osteoporos Int 22: 2175-2186, 2011.

12. Thomas PD, Campbell MJ, Kejariwal A, Mi H, Karlak B, Daverman R, Diemer K, Muruganujan A and Narechania A: PANTHER: A library of protein families and subfamilies indexed by function. Genome Res 13: 2129-2141, 2003.

13. Mi H, Guo N, Kejariwal A and Thomas PD: PANTHER version 6: Protein sequence and function evolution data with expanded representation of biological pathways. Nucleic Acids Res 35 (Database): D247-D252, 2007.

14. von Mering C, Jensen LJ, Snel B, Hooper SD, Krupp M, Foglierini M, Jouffre N, Huynen MA and Bork P: STRING: Known and predicted protein-protein associations, integrated and transferred across organisms. Nucleic Acids Res 33: D433-D437, 2005.

15. von Mering C, Jensen LJ, Kuhn M, Chaffron S, Doerks T, Krüger B, Snel B and Bork P: STRING 7 - recent developments in the integration and prediction of protein interactions. Nucleic Acids Res 35 (Database): D358-D362, 2007.

16. Duiker EW, Mom CH, de Jong S, Willemse PH, Gietema JA, van der Zee AG and de Vries EG: The clinical trail of TRAIL. Eur J Cancer 42: 2233-2240, 2006.

17. Mellier G, Huang S, Shenoy K and Pervaiz S: TRAILing death in cancer. Mol Aspects Med 31: 93-112, 2010.

18. Adams J: Development of the proteasome inhibitor PS-341. Oncologist 7: 9-16, 2002.

19. Melvin AT, Woss GS, Park JH, Waters ML and Allbritton NL: Measuring activity in the ubiquitin-proteasome system: From large scale discoveries to single cells analysis. Cell Biochem Biophys 67: 75-89, 2013.

20. Kubiczková L, Matějíková J, Sedlaříková L, Kryukov F, Hájek R and Sevčíková S: Proteasome inhibitors in treatment of multiple myeloma. Klin Onkol 26: 11-18, 2013 (In Czech).

21. Plowman JE: The proteomics of keratin proteins. J Chromatogr B Analyt Technol Biomed Life Sci 849: 181-189, 2007. 